
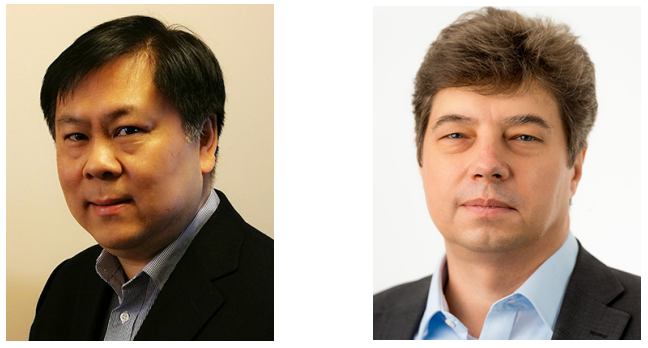

\title{
Editorial: Welcome to Advanced Photonics
}

Welcome to a new home for your best optics and photonics research. Advanced Photonics is a highly selective, open-access, international journal that publishes innovative research in all areas of optics and photonics, including fundamental and applied research. Photonics underpins the most impactful technologies of the twenty-first century, contributing and defining such diverse areas as information processing, internet and communications, imaging, sensing and security, healthcare and medicine, and quantum technology, among others. We will publish top-quality original papers, letters, and review articles, reflecting significant advances and breakthroughs in theoretical and experimental optics and photonics research in all areas, and novel applications with considerable potential. We most welcome interdisciplinary research articles demonstrating how photonics advances material science, biology, chemistry, and other disciplines.

Why do we need yet another optics journal? In recent years, the optics and photonics research community has achieved and reported many important ideas and breakthroughs. Indeed, there are quite a few top journals with high impact factors where we can publish. Advanced Photonics has been conceived to address the growing demand in the research community to be able to publish the best research results fast, and to make them visible to our peers and accessible to everyone.

In order to provide a broader community perspective, in addition to research letters, original articles, and reviews, we will publish news and commentaries written by leading experts in respective fields, highlighting scientific and technological discoveries and achievements, and explaining them to an interdisciplinary audience of researchers, to ensure that these new developments are appreciated fully. We will also publish the personal views of our authors who will explain how their new ideas emerged. We hope this will make an exciting mixture of scientific and general material for our readers.

We have been very lucky in assembling an outstanding editorial board of world-leading experts in their fields. They all know well themselves how frustrating it is to wait for a manuscript to be reviewed and published. In our journal, we will do our utmost to make this process as smooth and as fair as possible whilst maintaining the highest scientific quality. The editors aim to make Advanced Photonics a trusted source of groundbreaking research in optics and optical technologies.

We hope very much that Advanced Photonics will become a journal of choice for all innovative researchers and a must-read for the optics and photonics community. With the benefit of open access, we hope the journal will be on the reading list of everyone interested in new trends in photonic sciences. We are looking forward, with your help, to making Advanced Photonics a home for the best and most innovative research in optics and related disciplines.

Xiao-Cong (Larry) Yuan Co-Editor-in-Chief

Anatoly Zayats Co-Editor-in-Chief 